\title{
Spontaneous Cataractous Lens Extrusion in a Patient with Corneal Melting
}

\author{
Bader AlQahtani ${ }^{\mathrm{a}}$ Ahmad Abdelaal $^{\mathrm{a}}$ Ehab Karamallah $^{\mathrm{b}}$ \\ aOphthalmology Department, King AbdulAziz Medical City, Jeddah, Saudi Arabia; \\ ${ }^{b}$ Alsaggaf Eye Center, Jeddah, Saudi Arabia
}

\section{Keywords}

Cataract $\cdot$ Spontaneous extrusion $\cdot$ Cornea $\cdot$ Melt

\begin{abstract}
Purpose: The aim of this report is to describe an unusual case of cataractous lens extrusion through a diseased cornea in the absence of any history of trauma or surgery. Patient and Methods: An elderly female with a history of blepharitis, corneal vascularization, and scarring presented with foreign body sensation, mild ocular pain, and a brunescent cataractous lens extruding through an area of corneal melt of the right eye after being lost to follow-up. The patient was operated urgently and then referred to a specialized eye hospital for further management. Conclusion: In the setting of social neglect and poor compliance to treatment and appointments, consequences of common ophthalmic conditions can lead to serious vision and organ-threatening complications. These might require prompt medical and surgical attention.
\end{abstract}

\section{Introduction}

Spontaneous extrusion of implantable intraocular lenses has been reported in the literature $[1,2]$. We report a case of spontaneous hard brown cataractous lens extrusion through a severely diseased cornea. Urgent surgical management is highly recommended in these 
situations due to the potentially serious sight and organ threatening complications that may occur.

\section{Case Presentation}

A neglected 101-year-old female patient presented complaining of itchiness and foreign body sensation in both eyes. She was diabetic and hypertensive with no medical history of rheumatism or other autoimmune disease. Her last visit in July 2017 revealed best-corrected visual acuity of no light perception in the right eye and 20/100 in the left. Slit-lamp examination showed a moderate central corneal opacity with peripheral corneal vascularization, dense mature cataract, and a non-reactive pupil in the right eye. The left eye had mild central corneal opacity and was pseudophakic. Fundus visualization was not possible for the right eye, and a consequent B-scan confirmed a flat retina, while fundus examination of the left eye revealed dry, age-related macular degeneration. The patient was diagnosed as a case of bilateral posterior blepharitis with dry eyes, and an antibiotic/steroid ointment was prescribed along with heavy lubrication. Follow-up was given for possible extraction of her right eye cataract. The patient was lost to follow-up until September 2019 when she presented again with foreign body sensation and minimal ocular pain. Examination revealed no light perception with best-corrected visual acuity being the same as on her last visit. A hard, brown cataractous lens with a sharp edge was eroding through an area of central corneal melt with severe peripheral vascularization of the right eye (Fig. 1). The eye remained formed without evidence of hypotony. On further questioning, the patient denied any form of trauma. Subsequently, she was taken to the operating theater urgently to repair the corneal defect. Due to the unavailability of corneal grafts at that time, a rotating vascularized conjunctival flap was incised to cover the defect with interrupted 10-0 silk sutures. Thereafter, the patient was referred to a specialized eye hospital for further management.

\section{Discussion}

Few cases have been reported where implantable intraocular lenses have extruded through ocular tissues in the setting of ocular conditions such as scleromalacia perforans or following penetrating keratoplasty $[1,2]$. There is a single case report of crystalline lens displacement into the subconjunctival space through a suspected old globe penetration [3].

In contrast, our patient's virgin eye, without a history of preceding trauma or surgery, had previously demonstrated severe corneal scarring and vascularization. Regarding the corneal opacities, no reason could be identified, especially because the patient could not provide sufficient information on her medical history. Since the patient had been on antibiotic and steroid drops for a very long time (since July 2017) without doctor supervision or follow-up, this might have played a role. In the setting of social neglect with suboptimal medical care, corneal melting with repeated eye rubbing due to blepharitis might be the underlying causes leading to her unusual presentation where a brunescent cataractous lens was found extruding through corneal perforation.

The cataract extrusion was totally painless, which might have been due to the very sick, vascularized melted cornea we usually see in some patients with peripheral ulcerative keratitis who present with severe corneal thinning and impending perforation without pain. In addition, it might be explained by the long history of diabetes mellitus and senility. To the best 
of our knowledge, this is the first report of spontaneous cataractous lens extrusion with associated corneal pathology.

\section{Conclusion}

In conclusion, patients should be aware of the consequences of neglect, poor care, and noncompliance with medications or follow-up appointments. Ocular tissues are frail, and as long as the patient has predisposed corneal pathology, spontaneous cataractous lens and other serious complications can occur.

\section{Statement of Ethics}

This case report complies with the principles of the Declaration of Helsinki. Oral consent to publish this case has been obtained, but written consent has not been obtained. This report does not contain any personal identifying information.

\section{Disclosure Statement}

The authors have no conflicts of interest to declare.

\section{Funding Sources}

No funding was received.

\section{Author Contributions}

B.A. Collected the data, analyzed the ophthalmological findings, gave critical suggestions, and prepared the report.

A.A. revised and finalized the paper.

E.K. treated the patient, including surgery, and prepared the report.

All authors agree to be accountable for all aspects of the work. All authors attest that they meet the current ICMJE criteria for authorship.

\section{References}

1 Ahmed TY, Carrim ZI, Diaper CJ, Wykes WN. Spontaneous intraocular lens extrusion in a patient with scleromalacia secondary to herpes zoster ophthalmicus. J Cataract Refract Surg. 2007 May;33(5):925-6.

2 Cameron JD, Doughman DJ. Transcorneal extrusion of an intraocular lens. Ophthalmology. 1983 Apr;90(4):404-9.

3 Goel N. Displacement of crystalline lens into the sub-conjunctival space following periocular anesthesia. Saudi J Ophthalmol. 2018 Jul-Sep;32(3):257-60. 
Case Reports in
Ophthalmology

Case Rep Ophthalmol 2020;11:177-180

(c) 2020 The Author(s). Published by S. Karger AG, Basel www.karger.com/cop

AlQahtani et al.: Spontaneous Cataractous Lens Extrusion in a Patient with Corneal Melting

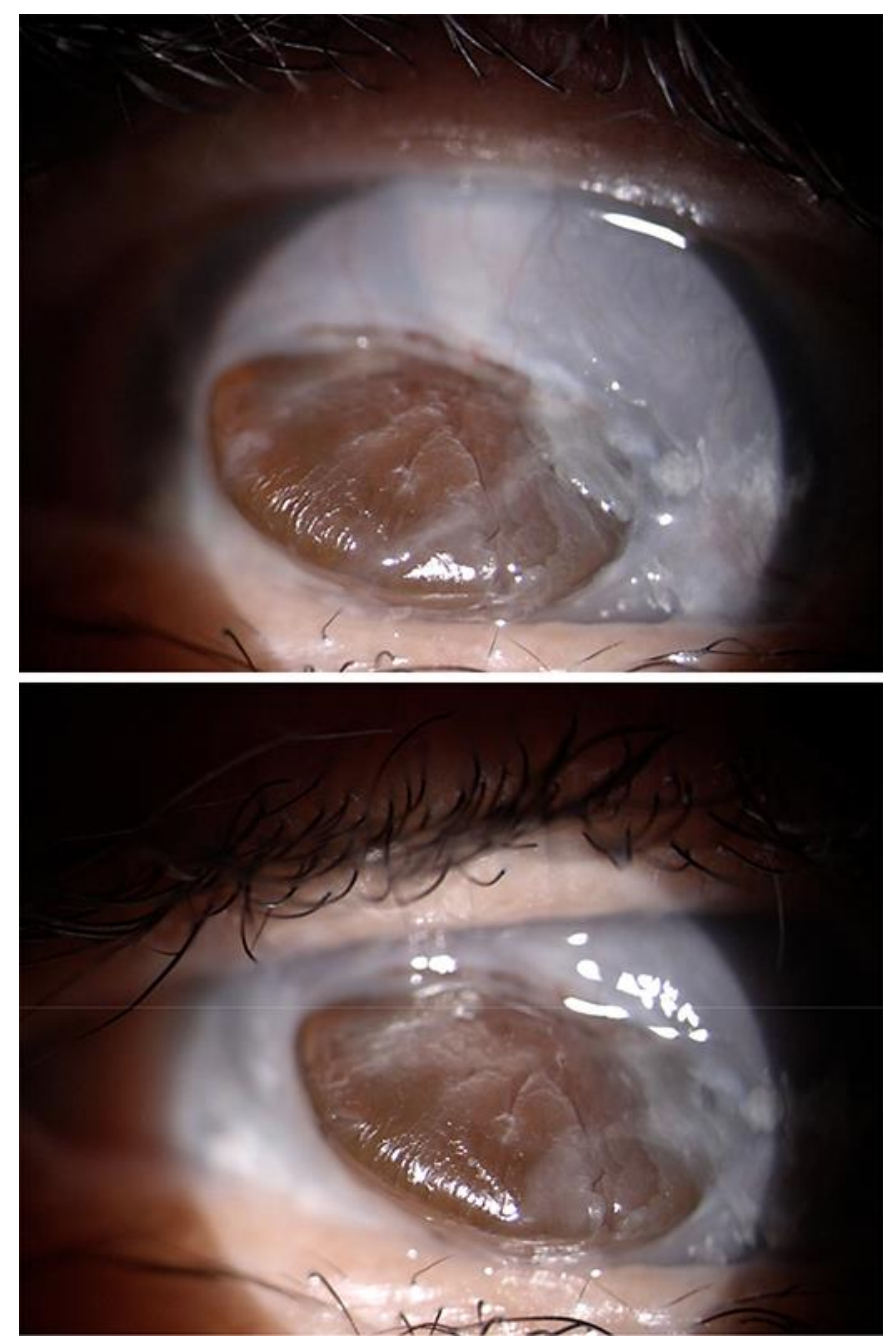

Fig. 1. Anterior segment photos of the brown cataract extruding through the cornea. 